\title{
CRITICAL MULTISCALE FLOW FOR INTERFACIAL SLIPPAGE IN MICROCHANNEL
}

\author{
Zhipeng Tang ${ }^{\mathrm{a}}$ and Yongbin Zhang ${ }^{\mathrm{b}, *}$ \\ ${ }^{a}$ College of Mechanical Engineering, Changzhou Vocational Institute of Mechatronic Technology, Changzhou, 213164, Jiangsu, China \\ ${ }^{b}$ College of Mechanical Engineering, Changzhou University, Changzhou, 213164, Jiangsu, China
}

\begin{abstract}
The critical flow rate through a micro/nano slit pore for starting the adsorbed layer-fluid or adsorbed layer-wall interfacial slippage is calculated by a multiscale scheme. There are the physical adsorbed layers on the channel walls and the intermediate continuum fluid which are respectively in noncontinuum and continuum flows. The flow factor approach model for nanoscale flow is used to simulate the adsorbed layer flow, and a continuum model describes the continuum fluid flow. The boundary between the adsorbed layer and the continuum fluid or the boundary between the adsorbed layer and the channel wall are treated as the interfacial slippage. The result is also compared with that when the adsorbed layer is treated as an immobile solid layer.
\end{abstract}

Keywords: Adsorbed layer; Flow; Interfacial slippage; Multiscale scheme; Micro/nano channel; Model

\section{INTRODUCTION}

Macrochannel flow has been well studied by conventional flow theories(Cho, 1982; Kawamura et al., 1986; Lemenand and Peerhossaini, 2002; Vallikivi et al., 2011), which neglect the effect of the very thin adsorbed layer on the wall surface. About half an century ago, nanochannel flow entered people's eyes. It possesses special phenomena including the fluid ordering to the wall surface, the rheological property variation across the channel height, and the interfacial slippage, which make the nanochannel flow essentially non-continuum and distinctly different from conventional continuum fluid flow (Gee et al., 1990; Jabbarzadeh et al., 1997; Joseph et al., 2006; Heinbuch and Fischer, 1989; Thompson and Robbins, 1990; Zhang, 2016). A nanochannel flow is typically the flow of the very thin layer physically adsorbed to the wall surfaces. There is a kind of microchannel flow which intervenes between macrochannel flow and nanochannel flow; This flow exists in microporous membrane filtration, biological tissues, micro transport and micro engineering etc. In this flow, the flow of the adsorbed layer on the wall surface is comparable to that of the continuum fluid intermediate between the two adsorbed layers; The effect of the adsorbed layer should depend on the ratio of the adsorbed layer thickness to the continuum fluid film thickness and the fluid-wall interaction strength. It is required to study the mechanism of this microchannel flow and find the way to efficiently improve the mass transfer in the related fields. Obviously, a multiscale scheme is required to simulate this microchannel flow. It should model both the non-continuum flow of the adsorbed layer and the continuum fluid flow.

In the past time, a lot of multiscale approaches were proposed for modeling such multiscale flows (Atkas and Aluru, 2002; Liu et al., 2007; Nie et al., 2004; Sun et al., 2010; Yang and Zheng, 2010; Yen et al., 2007); Normally, molecular dynamics simulation was used to model the flow of the layer adjacent to the wall surface, and a continuum theory modeled the intermediate continuum fluid flow. The obvious shortcoming of the conventional multiscale schemes is that they should take long computational time for a long channel. Recently, a new multiscale approach was proposed for simulating the above mentioned multiscale channel flow (Zhang, 2019a, 2020). It describes the flow of the adsorbed layer by the flow factor approach model for nanoscale flow and describes the intermediate continuum fluid flow by a continuum model. This multiscale approach can give the closed-form explicit flow equations respectively for the flows of the adsorbed layer and the continuum fluid. Its obvious advantage is that it can very efficiently solve an engineering problem with practical long channels.

Based on this advanced multiscale scheme, the present paper presents the analytical and computational results of the critical total multiscale flow rate through a micro slit pore driven by the pressure for starting the interfacial slippage on the adsorbed layer-fluid interface or on the adsorbed layer-wall surface interface, when different fluid-wall interactions are considered. Once the flow in the channel exceeds this critical value, the interfacial slippage will occur. The study should be of

*Corresponding author.Email: engmech1@sina.com 
interest to the design of microchannel flow for improving the flow rate by using the interfacial slippage.

\section{MODELED MICROCHANNEL FLOW}

Figure 1(a) shows the modeled pressure driven microchannel flow where the thickness of the physical adsorbed layer on the channel wall is comparable to the film thickness of the intermediate continuum fluid. The adsorbed layer may participate in the flow more or less depending on the fluid-wall interaction. For carrying out a feasible multiscale analysis for the flow in Fig. 1(a), the physical adsorbed layer is equivalently shown as in Fig. 1(b). Then, the adsorbed layer flow is described by the flow factor approach model for nanoscale flow (Zhang, 2019a,2020), and the flow of the intermediate continuum fluid is described by a continuum flow theory. In a normal channel flow, the interfacial slippage may first occur on the adsorbed layer-fluid interface instead of on the adsorbed layer-wall surface interface because of the weaker strength of the adsorbed layer-fluid interface (Rozeanu and Snarsky, 1977). However, when the channel wall is covered with a hydrophobic coating, the interfacial slippage may occur on the adsorbed layer-wall surface interface. In the present study, these two cases are considered. In Fig. 1(b), the pressure gradient is assumed as negative so that the flow is from the left-hand side to the right-hand side. The used coordinates are also shown in Fig. 1(b).

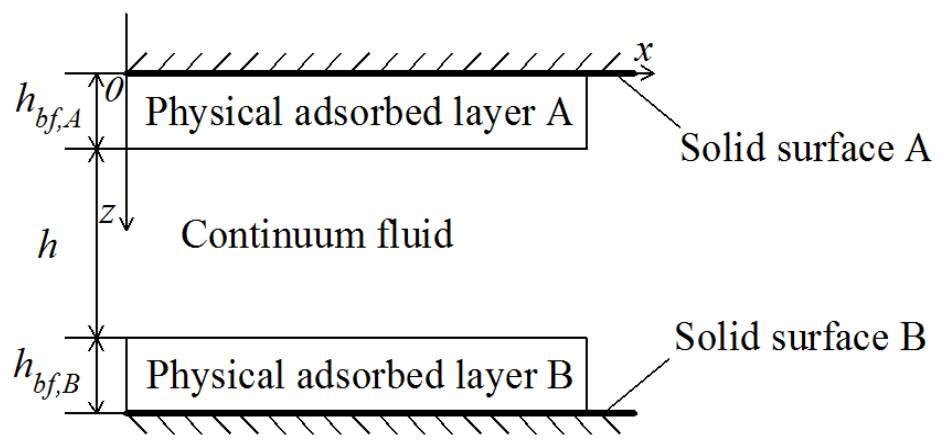

(a)

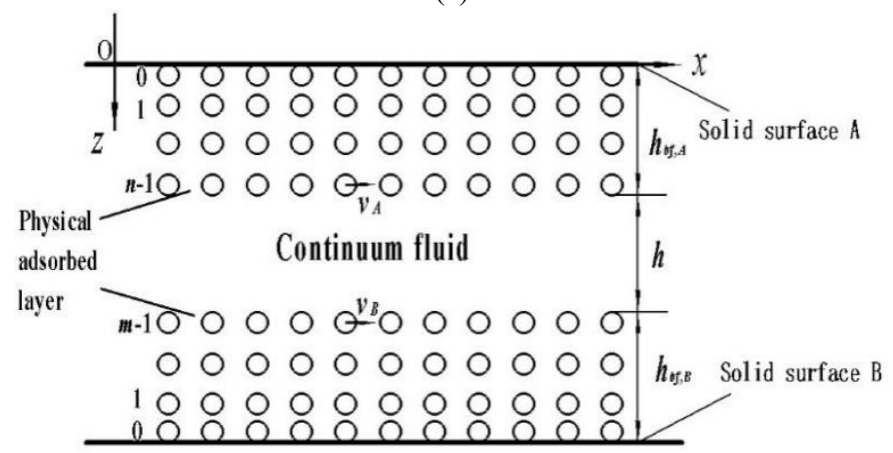

(b)

Fig. 1 Modeled multiscale flow in a micro slit pore (Zhang, 2019a, 2020).

\section{ANALYTICAL RESULTS}

\subsection{When the interfacial slippage occurs on the adsorbed layer-wall surface interface}

In this case, no slippage occurs on the adsorbed layer-fluid interface. When the adsorbed layer is treated as a flowing layer, the detailed analysis for the multiscale flow in Fig. 1 (b) has been shown by Zhang (2020). Here are only presented the derived results.
Both the interfacial shear stresses $\tau_{a}$ and $\tau_{b}$ respectively on the upper and lower adsorbed layer-wall surface interfaces should be equal to the adsorbed layer-wall surface interfacial shear strength $\tau_{s, b-w}$. Thus (Zhang, 2020):

$\tau_{a}=\frac{\bar{u}_{b}-\bar{u}_{a}}{\frac{2 \eta}{h} \sum_{j=1}^{n-1} \frac{\Delta_{j-1}}{\eta_{l i n e, j-1}}+1} \frac{\eta}{h}-\frac{\partial p}{\partial x}\left(\frac{1}{2} h+D n\right)+\frac{\partial p}{\partial x} D=\tau_{s, b-w}$

and

$$
\begin{aligned}
\tau_{b} & =-\frac{\left(\bar{u}_{b}-\bar{u}_{a}\right)\left(\frac{\frac{\eta}{h} \sum_{j=1}^{n-1} \frac{\Delta_{j-1}}{\eta_{\text {line }, j-1}}+1}{\frac{2 \eta}{n} \sum_{j=1}^{n-1} \frac{\Delta_{j-1}}{\eta_{\text {line }, j-1}}+1}\right)-\frac{\partial p}{\partial x}\left(\frac{1}{2} h+D n\right) \sum_{j=1}^{n-1} \frac{\Delta_{j-1}}{\eta_{\text {line }, j-1}}}{\sum_{j=1}^{n-1} \frac{\Delta_{j-1}}{\eta_{\text {line }, j-1}}}-\frac{\partial p}{\partial x} D \\
=-\tau_{s, b-w} &
\end{aligned}
$$

Solving the coupled equations (1) and (2) gives that $\bar{u}_{a}=\bar{u}_{b}$ and:

$$
\frac{\partial p}{\partial x}=-\frac{\tau_{s, b-w}}{\frac{1}{2} h+D(n-1)}
$$

When the coupled channel wall surfaces are the same, the upper and lower adsorbed layers are the same. For this case, according to the analysis developed by Zhang (2020), by using Eq.(3), the critical dimensionless total volume flow rate per unit channel width through the channel for starting the interfacial slippage is:

$$
\begin{aligned}
& Q_{v, c r, 1}=-\frac{1}{\frac{1}{2}+\lambda_{b f}-\frac{q_{0}-q_{0}^{n} \Delta_{n-2}}{q_{0}^{n-1}-q_{0}^{n} h}-\frac{D}{h}}\left\{\frac{F_{1} \lambda_{b f}}{6 C_{y}}-\frac{1}{12 \lambda_{b f}^{2}}-\left(1+\frac{1}{2 \lambda_{b f}}-\frac{q_{0}-q_{0}^{n}}{q_{0}^{n-1}-q_{0}^{n}}\right.\right. \\
& \left.\left.\times \frac{\Delta_{n-2}}{h_{b f}}\right) \frac{\varepsilon \lambda_{b f}}{C_{y}\left(1+\frac{\Delta x}{D}\right)}+\frac{1}{C_{y} \lambda_{b f}^{2}}\left[\frac{F_{2} \lambda_{b f}^{2}}{6}-\frac{\lambda_{b f}}{1+\frac{\Delta x}{D}}\left(\frac{1}{2}+\lambda_{b f}-\frac{q_{0}-q_{0}^{n}}{q_{0}^{n-1}-q_{0}^{n}} \frac{\Delta_{n-2}}{h}\right)\right]\right\}
\end{aligned}
$$

where $Q_{v, c r, 1}=q_{v, c r, 1} \eta /\left(\tau_{s, b-w} h_{b f}^{2}\right), \quad C_{y}=\eta_{b f}^{e f f} / \eta, \quad \lambda_{b f}=h_{b f} / h$, $F_{1}=\eta_{b f}^{e f f}\left(12 D^{2} \psi+6 D \phi\right) / h_{b f}^{3} \quad, \quad F_{2}=6 \eta_{b f}^{e f f} D(n-1)\left(l \Delta_{l-1} /\right.$ $\left.\eta_{\text {line }, l-1}\right)_{a v r, n-1} / h_{b f}^{2} \quad, \quad \varepsilon=(2 D I+I I) /\left[h_{b f}(n-1)\left(\Delta_{l} /\right.\right.$ $\left.\left.\eta_{\text {line }, l}\right)_{\text {avr }, n-1}\right], i\left(\Delta_{l} / \eta_{\text {line }, l}\right)_{\text {avr }, i}=\sum_{j=1}^{i} \Delta_{j-1} / \eta_{\text {line }, j-1}, i\left(l \Delta_{l-1} /\right.$ $\left.\eta_{\text {line }, l-1}\right)_{a v r, i}=\sum_{j=1}^{i} j \Delta_{j-1} / \eta_{\text {line }, j-1} \quad, \quad \psi=\sum_{i=1}^{n-1} i\left(l \Delta_{l-1} /\right.$ $\left.\eta_{\text {line }, l-1}\right)_{a v r, i}$, and $\phi=\sum_{i=0}^{n-2}\left[i\left(l \Delta_{l-1} / \eta_{\text {line }, l-1}\right)_{a v r, i}+(i+1)\left(\Delta_{l} /\right.\right.$ $\left.\left.\eta_{\text {line }, l}\right)_{a v r, i+1}\right] \Delta_{i}$. Here, $\quad \eta_{b f}^{\text {eff }}=D h_{b f} /\left[(n-1)\left(D+\Delta_{x}\right)\left(\Delta_{l} /\right.\right.$ $\left.\left.\eta_{\text {line,l }}\right)_{\text {avr }, n-1}\right]$.

\subsection{When the interfacial slippage occurs on the adsorbed layer-fluid interface}

In this case, no slippage occurs on the adsorbed layer-wall surface interface. When the adsorbed layer is treated as a flowing layer, the critical dimensionless total volume flow rate per unit channel width through the channel for starting the interfacial slippage is (Zhang, 2019a):

$$
\begin{aligned}
& Q_{v, c r, 2}=\frac{\varepsilon}{\left(1+\frac{\Delta x}{D}\right) C_{y}}+\frac{2 \varepsilon \lambda_{b f}}{\left(1+\frac{\Delta x}{D}\right) C_{y}}\left(1-\frac{q_{0}-q_{0}^{n}}{q_{0}^{n-1}-q_{0}^{n}} \frac{\Delta_{n-2}}{h_{b f}}\right)-\frac{\lambda_{b f} F_{1}}{3 C_{y}}+\frac{1}{6 \lambda_{b f}^{2}} \\
& +\frac{1}{C_{y} \lambda_{b f}}\left\{\frac{1}{1+\frac{\Delta x}{D}}-2 \lambda_{b f}\left[\frac{F_{2}}{6}-\left(1-\frac{q_{0}-q_{0}^{n}}{q_{0}^{n-1}-q_{0}^{n}} \frac{\Delta_{n-2}}{h_{b f}}\right) \frac{1}{1+\frac{\Delta x}{D}}\right]\right\}
\end{aligned}
$$

where $Q_{v, c r, 2}=q_{v, c r, 2} \eta /\left(\tau_{s, b-f} h_{b f}^{2}\right)$. 
When the adsorbed layer is treated as an immobile solid layer, the critical dimensionless total volume flow rate per unit channel width through the channel for starting the interfacial slippage is (Zhang, 2019b):

$$
Q_{v, s, c r}=\frac{1}{6 \lambda_{b f}^{2}}
$$

where $Q_{v, s, c r}=q_{v, s, c r} \eta /\left(\tau_{s, b-f} h_{b f}^{2}\right)$.

\section{CALCULATION}

In the present calculation, $\Delta \mathrm{x} / \mathrm{D}=\Delta_{n-2} / \mathrm{D}=0.15$ and $D=0.5 \mathrm{~nm}$. It was taken that $\eta_{\text {line }, i} / \eta_{\text {line }, i+1}=q_{0}^{m}$. It was formulated that (Zhang, 2014):

$\mathrm{C}_{y}\left(H_{b f}\right)=\frac{\eta_{b f}^{e f f}\left(H_{b f}\right)}{\eta}=a_{0}+\frac{a_{1}}{H_{b f}}+\frac{a_{2}}{H_{b f}^{2}}$

where $H_{b f}=h_{b f} / h_{c r, b f}, h_{c r, b f}$ is a critical thickness, and $a_{0}, a_{1}$ and $a_{2}$ are respectively constant and their values are shown in Table 1.

Table 1 Fluid viscosity data for different fluid-wall interactions (Zhang, 2014)

\begin{tabular}{|c|c|c|c|}
\hline Parameter & $\mathrm{a}_{0}$ & $\mathrm{a}_{1}$ & $\mathrm{a}_{2}$ \\
\hline Interaction & 1.8335 & -1.4252 & 0.5917 \\
\hline Mtrong & 1.0822 & -0.1758 & 0.0936 \\
\hline Wedium & 0.9507 & 0.0492 & $1.6447 \mathrm{E}-4$ \\
\hline
\end{tabular}

The weak, medium and strong fluid-wall interactions considered respectively have the following characteristic parameter values:

Weak interaction: $m=0.5, n=3, q_{0}=1.03, \quad h_{c r, b f}=7 \mathrm{~nm}$

Medium interaction: $m=1.0, n=5, q_{0}=1.1, h_{c r, b f}=20 \mathrm{~nm}$

Strong interaction: $m=1.5, n=8, q_{0}=1.2, \quad h_{c r, b f}=40 \mathrm{~nm}$

\section{RESULTS}

Figure 2(a) shows the calculated values of $Q_{v, c r, 1}, Q_{v, c r, 2}$ and $Q_{v, s, c r}$ for different $\lambda_{b f}$ when the fluid-wall interaction is weak. For the same $\lambda_{b f}$, the value of $Q_{v, c r, 2}$ is close to but a bit greater than that of $Q_{v, c r, 1}$. This may indicate that if the wall surface is designed as hydrophobic so that the fluid-wall interaction is weak, the adsorbed layer-wall surface interfacial slippage may be more easily generated than the adsorbed layer-fluid interfacial slippage in a microchannel flow, as the interfacial shear strength $\tau_{s, b-w}$ on the adsorbed layer-wall surface interface can be considerably smaller than the interfacial shear strength $\tau_{s, b-f}$ on the adsorbed layer-fluid interface. A sufficient dimensional volume flow rate per unit channel width through the channel may cause the interfacial slippage on the adsorbed layer-wall surface interface to first occur, since the dimensional critical volume flow rate $q_{v, c r, 1}$ through the channel for the occurrence of the adsorbed layer-wall surface interfacial slippage can be considerably lower than the dimensional critical volume flow rate $q_{v, c r, 2}$ through the channel for the occurrence of the adsorbed layer-fluid interfacial slippage. When $\lambda_{b f}<0.1$, for the same $\lambda_{b f}$, the value of $Q_{v, c r, 2}$ is considerably larger than that of $Q_{v, s, c r}$ especially for low $\lambda_{b f}$ values. This may indicate that when calculating the dimensional critical volume flow rate through the channel for the adsorbed layer-fluid interfacial slippage for the weak fluid-wall interaction, the adsorbed layer should be treated as a flowing layer even for low values of $\lambda_{b f}$. The case is the same for a high $\lambda_{b f}$. When $\lambda_{b f}$ is as high as over 0.1, for a given $\lambda_{b f}$, the values of $Q_{v, c r, 1}$ and $Q_{v, c r, 2}$ are much closer and nearly overlaid. The curves for $Q_{v, c r, 1}$ and $Q_{v, c r, 2}$ in Fig. 2(a) for the entire range of $\lambda_{b f}$ indicate the possibility of generating the adsorbed layer-wall surface interfacial slippage in a microchannel flow by designing the hydrophobic channel wall surfaces. According to the relation $q_{v, c r, 1}=Q_{v, c r, 1} \tau_{s, b-w} h_{b f}^{2} / \eta$, low values of $Q_{v, c r, 1}, \tau_{s, b-w}$ and $h_{b f}$ but a high value of $\eta$ results in a low value of $q_{v, c r, 1}$, which is very beneficial for the fluid transportation through the channel, as the adsorbed layer-wall surface interfacial slippage is easily generated and thus significantly improves the flow rate through the channel even in the condition of a small flow rate through the channel.

Figure 2(b) shows the calculated values of $Q_{v, c r, 1}, Q_{v, c r, 2}$ and $Q_{v, s, c r}$ for different $\lambda_{b f}$ when the fluid-wall interaction is mediumlevel. The values of $Q_{v, c r, 1}, Q_{v, c r, 2}$ and $Q_{v, s, c r}$ in Fig. 2(b) are much closer to each other than in Fig. 2(a). For a medium fluid-wall interaction as popular in normal microchannel flows, the interfacial shear strength $\tau_{s, b-w}$ on the adsorbed layer-wall surface interface may be considerably larger than the interfacial shear strength $\tau_{s, b-f}$ on the adsorbed layerfluid interface, according to Fig. 2(b), the adsorbed layer-fluid interfacial slippage may be more easily generated than the adsorbed layer-wall surface interfacial slippage in a microchannel flow, as the dimensional critical volume flow rate $\left(q_{v, c r, 2}\right)$ through the channel for the adsorbed layer-fluid interfacial slippage is considerably smaller than that $\left(q_{v, c r, 1}\right)$ for the adsorbed layer-wall surface interfacial slippage. This fits the argument by Rozeanu and Snarsky (Rozeanu and Snarsky, 1977) that in a normal hydrodynamic contact the interfacial slippage would first occur on the boundary layer-fluid interface because of the entropy discontinuity there. Figure 2(b) shows that for a medium fluid-wall interaction, when calculating the dimensional critical volume flow rate through the channel for the adsorbed layer-fluid interfacial slippage, the adsorbed layer should still be treated as a flowing layer in the entire range of $\lambda_{b f}$, as there are still obvious divergences between $Q_{v, c r, 2}$ and $Q_{v, s, c r}$ for given $\lambda_{b f}$.

Figure 2(c) shows that for a strong fluid-wall interaction, the calculated values of $Q_{v, c r, 1}, Q_{v, c r, 2}$ and $Q_{v, s, c r}$ are nearly the same for a given $\lambda_{b f}$. This may indicate that for a strong fluid-wall interaction, the dimensional critical volume flow rate $\left(q_{v, c r, 1}\right)$ through the channel for the adsorbed layer-wall surface interfacial slippage is much greater than that $\left(q_{v, c r, 2}\right)$ for the adsorbed layer-fluid interfacial slippage, as the interfacial shear strength $\tau_{s, b-w}$ on the adsorbed layer-wall surface interface is much greater than the interfacial shear strength $\tau_{s, b-f}$ on the adsorbed layer-fluid interface. In this case, the interfacial slippage should first occur on the adsorbed layer-fluid interface depending on the value of $q_{v, c r, 2}$. Figure 2(c) shows that for a strong fluid-wall interaction, when calculating the dimensional critical volume flow rate through the channel for the adsorbed layer-fluid interfacial slippage, the adsorbed layer can be treated as an immobile solid layer in the entire range of $\lambda_{b f}$, as the curves for $Q_{v, c r, 2}$ and $Q_{v, s, c r}$ are nearly overlaid.

Figures 2(a)-(c) show that with the increase of $\lambda_{b f}$, both the values of $Q_{v, c r, 1}$ and $Q_{v, c r, 2}$ are rapidly reduced when $\lambda_{b f}<0.1$. This shows the very significant effect of reducing the intermediate continuum fluid film thickness $h$ on dropping the dimensional critical volume flow rates through the channel for the adsorbed layer-wall surface or the adsorbed layer-fluid interfacial slippage when $\lambda_{b f}<0.1$. A narrow channel may thus easily result in the interfacial slippage even with a very modest flow rate through the channel. However, when $\lambda_{b f}>0.1$, both the values of $Q_{v, c r, 1}$ and $Q_{v, c r, 2}$ are slowly varied with $\lambda_{b f}$. In this case, the thickness $h_{b f}$ of the adsorbed layer is on the same scale with 
the intermediate continuum fluid film thickness $h$, and a multiscale analysis is required for calculating $Q_{v, c r, 1}$ and $Q_{v, c r, 2}$ when the fluidwall interaction is weak or medium-level.

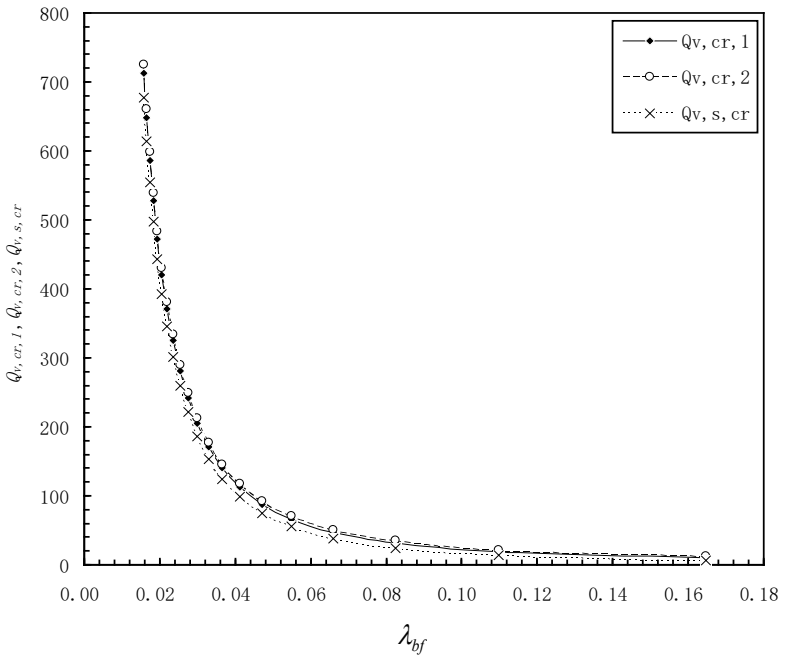

(a) For the weak fluid-wall interaction

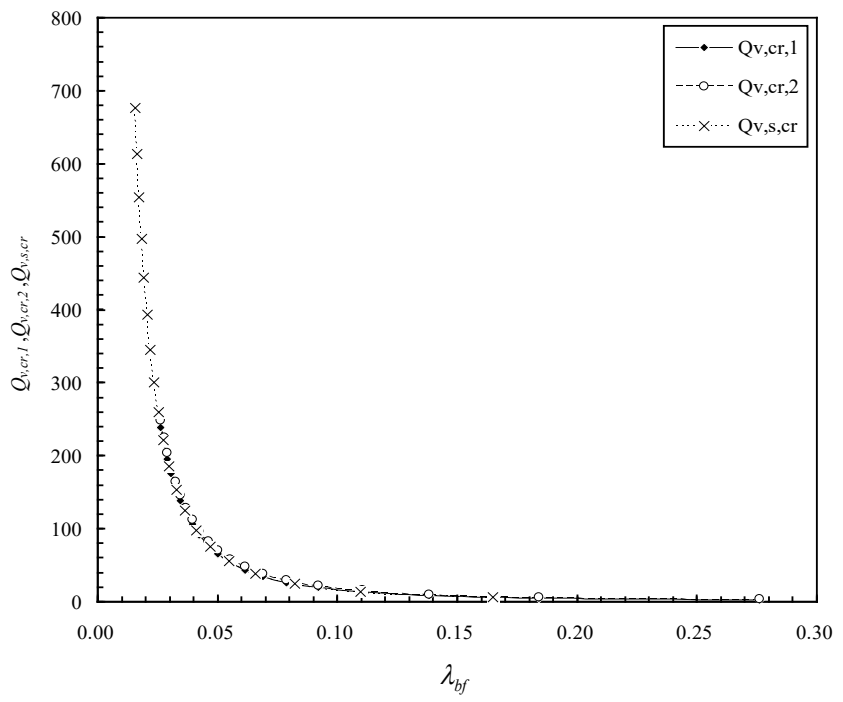

(b) For the medium fluid-wall interaction

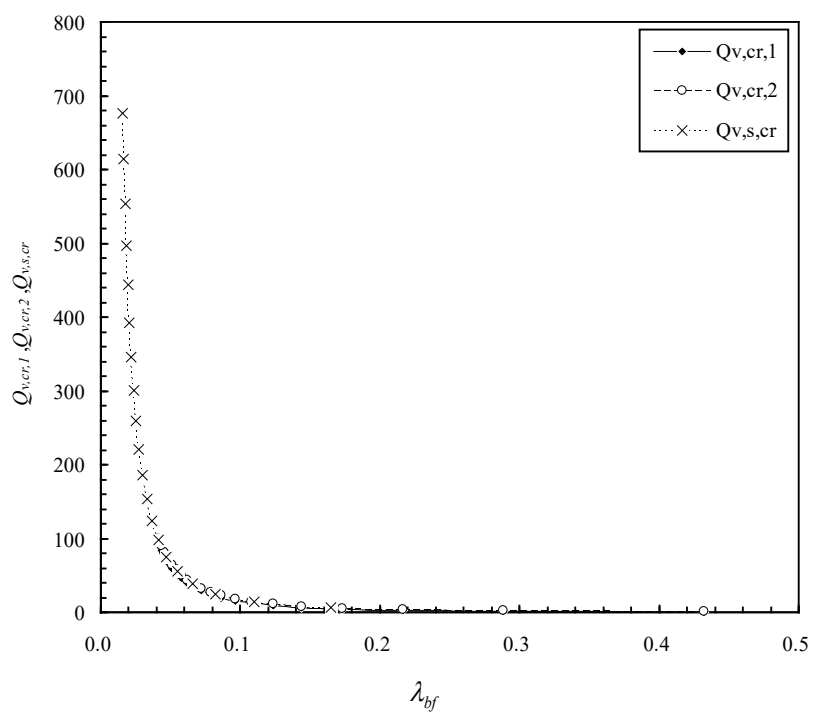

(c) For the strong fluid-wall interaction

Fig. 2 Values of $Q_{v, c r, 1}, Q_{v, c r, 2}$ and $Q_{v, s, c r}$.

\section{CONCLUSIONS}

The multiscale analytical results are presented for the critical volume flow rates per unit channel width through the channel respectively for starting the interfacial slippage on the adsorbed layer-fluid interface and on the adsorbed layer-wall surface interface in a pressure driven multiscale flow in a micro slit pore. In this flow, the thickness of the adsorbed layer on the channel wall is comparable to the film thickness of the continuum fluid intermediate between the two adsorbed layers. The flow factor approach model for nanoscale flow is used to describe the adsorbed layer flow, and a continuum model describes the intermediate continuum fluid flow. The calculation results have been obtained for different fluid-wall interactions and the conclusions are drawn as follows:

(1) When the channel wall surface is designed as hydrophobic so that the fluid-wall interaction is weak, the interfacial slippage may first occur on the adsorbed layer-wall surface interface instead of on the adsorbed layer-fluid interface, as the required dimensional critical volume flow rate through the channel for the adsorbed layer-wall surface interfacial slippage is considerably smaller than that for the adsorbed layer-fluid interfacial slippage.

(2) When the fluid-wall interaction is medium-level or strong, the interfacial slippage may first occur on the adsorbed layer-fluid interface, as the required dimensional critical volume flow rate through the channel for the adsorbed layer-fluid interfacial slippage is considerably smaller than that for the adsorbed layer-wall surface interfacial slippage.

(3) When the fluid-wall interaction is weak or medium-level, a multiscale analysis is required for calculating the dimensional critical volume flow rates through the channel respectively for the adsorbed layer-fluid interfacial slippage or the adsorbed layer-wall surface interfacial slippage, by treating the adsorbed layer as a flowing layer. When the fluid-wall interaction is strong, the adsorbed layer can be treated as an immobile solid layer in this calculation.

(4) A narrow channel easily results in the interfacial slippage even with a very modest flow rate through the channel.

\section{NOMENCLATURE}

$a_{0}, a_{1}, a_{2}$ constant, Eq.(7)

$D \quad$ fluid molecule diameter

$h \quad$ thickness of the intermediate continuum fluid

$h_{c r, b f} \quad$ critical film thickness

$h_{b f, A}, h_{b f, B}$ thicknesses of the physical adsorbed layers on the coupled channel walls respectively

$h_{b f} \quad$ thickness of either of the adsorbed layers on the channel walls, $h_{b f, A}$ or $h_{b f, B}$

$i, j \quad$ order numbers of the fluid molecule across the adsorbed layer thickness respectively, Fig. 1(b)

$m \quad$ positive index

$n \quad$ equivalent number of the fluid molecules across the adsorbed layer thickness

$p \quad$ fluid film pressure

$q_{v, c r, 1}$ dimensional critical volume flow rate per unit channel width through the channel for starting the adsorbed layer-wall surface interfacial slippage

$q_{v, c r, 2}$ dimensional critical volume flow rate per unit channel width through the channel for starting the adsorbed layer-fluid interfacial slippage

dimensional critical volume flow rate per unit channel width through the channel for starting the adsorbed layer-fluid interfacial slippage based on the solid layer assumption

$Q_{v, c r, 1}$ dimensionless critical volume flow rate per unit channel width through the channel for starting the adsorbed layer-wall 
surface interfacial slippage

$Q_{v, c r, 2}$ dimensionless critical volume flow rate per unit channel width through the channel for starting the adsorbed layer-fluid interfacial slippage

$Q_{v, s, c r} \quad$ dimensionless critical volume flow rate per unit channel width through the channel for starting the adsorbed layer-fluid interfacial slippage based on the solid layer assumption

$\bar{u}_{a} \quad$ velocity of the adsorbed layer on the upper channel wall surface

$\bar{u}_{b} \quad$ velocity of the adsorbed layer on the lower channel wall surface

$x, z \quad$ coordinates

$\eta \quad$ fluid bulk viscosity

$\tau_{s, b-f} \quad$ shear strength of the adsorbed layer-fluid interface

$\tau_{s, b-w} \quad$ shear strength of the adsorbed layer-wall surface interface

$\Delta x \quad$ separation between the neighboring fluid molecules in the flow direction

$\Delta_{j-1}, \eta_{\text {line,j-1 }}$ separation and local viscosity between the $j^{\text {th }}$ and $(j-1)^{t h}$ fluid molecules across the adsorbed layer thickness respectively

$\eta_{b f}^{e f f} \quad$ effective viscosity of the adsorbed layer

\section{REFERENCES}

Atkas, O., Aluru, N. R., 2002, "A combined continuum/DSMC technique for multiscale analysis of microfluidic filters," J. Comput. Phys, 178,342-372.

https://doi.org/10.1006/jcph.2002.7030

Cho, Y. I., 1982, "Non-Newtonian fluids in circular pipe flow," Adv. Heat Transf., 15, 59-141.

https://doi.org/10.1016/S0065-2717(08)70173-4

Gee, M. L., McGuiggan, P. M., Israelachvilli, J. N., 1990, "Liquid to solidlike transitions of molecularly thin films under shear," J. Chem. Phys., 93, 1895.

https://doi.org/10.1063/1.459067

Heinbuch, U., Fischer, J., 1989, "Liquid flow in pores: Slip, no-slip, or multilayer sticking," Phys. Rev. A, 40, 1144.

https://doi.org/10.1103/PhysRevA.40.1144

Jabbarzadeh, A., Atkinson, J. D., Tanner, R. I.,1997, "Rheological properties of thin liquid films by molecular dynamics simulations," $J$. Non-Newtonian Fluid Mech., 69, 169-193.

http://dx.doi.org/10.1016//S0377-0257(96)01520-0

Joseph, P., Cottin-Bizonne, C., Benoît, J. M., Ybert, C., Journet, C., Tabeling, P., Bocquet, L., 2006, "Slippage of water past superhydrophobic carbon nanotube forests in microchannels," Phys. Rev. Lett., 97, 156104.

https://doi.org/10.1103/PhysRevLett.97.156104

Kawamura, T., Takami, H., Kuwahara, K., 1986, "Computation of high Reynolds number flow around a circular cylinder with surface roughness," Fluid Dyn. Res., 1, 145-150.

https://doi.org/10.1016/0169-5983 (86)90014-6

Lemenand, T., Peerhossaini, H., 2002, "A thermal mode for prediction of the Nusselt number in a pipe with chaotic flow," Appl. Therm. Eng., 22, 1717-1730.

\section{https://doi.org/10.1016/s1359-4311(02)00075-3}

Liu, J., Chen, S., Nie, X., Robbins, M. O., 2007, “A continuum-atomistic simulation of heat transfer in micro- and nano- flows," J. Comput. Phys., 227, 279-291.

http://dx.doi.org/10.1016/j.jcp.2007.07.014

Nie, X. B., Chen, S., Robbins, M. O., 2004, “A continuum and molecular dynamics hybrid method for micro- and nano- fluid flow," J. Fluid Mech., 500, 55-64.

http://dx.doi.org/10.1017/s0022112003007225

Rozeanu, L., Snarsky, L., 1977, “The unusual behavior of a lubricant boundary layer," Wear, 43, 117-126.

https://doi.org/10.1016/0043-1648(77)90047-3

Sun, J., He, Y., Tao, W. Q., 2010, "Scale effect on flow and thermal boundaries in micro-/nano- channel flow using molecular dynamicscontinuum hybrid simulation method," Int. J. Num. Meth. Eng., 81, 207228.

http://dx.doi.org/10.1002/nme.2683

Thompson, P. A., Robbins, M. O., 1990, "Shear flow near solids: Epitaxial order and flow boundary conditions," Phys. Rev. A, 41, 6830. https://doi.org/10.1103/PhysRevA.41.6830

Vallikivi, M., Hultmark, M., Bailey, S. C. C., Smits, A. J., 2011, "Turbulence measurements in pipe flow using a nanoscale thermal anemometry probe," Exp. Fluids, 51, 1521-1527.

https://doi.org/10.1007/s00348-011-1165-4

Yang, X., Zheng, Z. C., 2010, "Effects of channel scale on slip length of flow in micro/nano channels," ASME J. Fluids Eng., 132, 061201. http://dx.doi.org/10.1115/1.4001619

Yen, T. H., Soong, C. Y., Tzeng, P. Y., 2007, "Hybrid molecular dynamics-continuum simulation for nano/mesoscale channel flows," Microfluid. Nanofluid., 3, 665-675. http://dx.doi.org/10.1007/s10404-007-0154-7

Zhang, Y. B., 2014, "Lubrication analysis for a line contact covering from boundary lubrication to hydrodynamic lubrication: Part I- Micro contact results," J. Comput. Theor. Nanosci., 11, 62-70.

https://doi.org/10.1166/jetn.2014.3336

Zhang, Y. B., 2016, "The flow equation for a nanoscale fluid flow," Int. J. Heat Mass Transf., 92, 1004-1008.

https://doi.org/10.1016/i.ijheatmasstransfer.2015.09.008

Zhang, Y. B., 2019a, "Multiscale flow in a micro slit pore with the adsorbed layer-fluid interfacial slippage," Appl. Math. Mod., submitted.

Zhang, Y. B., 2019b, "Influence of the fluid-wall interaction on multiscale flow through a micro slit pore considering the adsorbed layerfluid interfacial slippage," Front. Heat Mass Transf., 13, 27. http://dx.doi.org/10.5098/hmt.13.27

Zhang, Y. B., 2020, "Modeling of flow in a very small surface separation," Appl. Math. Mod., 82, 573-586. https://doi.org/10.1016/j.apm.2020.01.069. 